\title{
Masking-level differences and the form of the psychometric function'
}

\author{
JAMES P. EGAN, ${ }^{2}$ WILLIAM A. LINDNER, ${ }^{3}$ AND DENNIS MCFADDEN4 \\ INDIANA UNIVERSITY
}

\begin{abstract}
Functions showing the relation between-the detectability and the energy of a signal were determined for various interaural phase conditions. The empirical relation between $d$ and signal energy $E$ is approximately $d^{\prime}=m\left(E / N_{o}\right)^{k}$ where $m$ and $k$ are constants for a particular function. The data show that $k$ is fairly constant for a particular $O$ and that $m$ depends upon the interaural condition. That is, the various psychometric functions for a given $O$ all had the same form, independent of location. Therefore, the magnitude of the $M L D=(10 / k) \log \left(m_{i} / m_{r}\right)$, where $m_{i}$ is the constant for condition $i$, and $m_{r}$ corresponds to a reference condition. Consequently, an $M L D$ is relatively independent of the level of performance that is chosen for the determination of that $M L D$.
\end{abstract}

The detectability of an auditory signal that is presented in noise may be increased markedly by an appropriate change in the interaural relations of the signal or of the noise. The effects are so large that the enhanced detectability, or "release from masking," is typically measured, not as an increase in the detectability of the signal, but rather, as the change in the signal energy that is necessary in order to maintain the listener's performance at a constant level. This change in signal energy, expressed in decibels, is called a "masking-level difference," or MLD. The investigations of Licklider (1948), Hirsh (1948), and Hirsh and Webster (1949), mark the beginnings of this line of auditory research, and since the publication of these early studies, there has been a steady increase in our knowledge of binaural masking phenomena. In particular, Jeffress and his coworkers (e.g., Jeffress et al, 1952, 1956; Blodgett et al, 1958; Robinson \& Jeffress, 1963; Langford \& Jeffress, 1964) have made many significant contributions to this area of audition. Recently, Durlach (1963), Green (1966a), and others have developed theoretical approaches to these phenomena different from that developed by Jeffress, and these various models of binaural masking have contributed to an increased interest in MLDs.

As an example of a masking-level difference, suppose that the "masked absolute threshold" is determined for a sinusoidal signal when it and a continuous masking noise are presented to the right ear only, a condition called Nm-Sm. Next, the noise waveform that is presented to the right ear is presented simultaneously to the left ear, so that the instantaneous sound pressure of the noise in the left ear is the same as that in the right ear. With this perfectly correlated "binaural noise," the masked threshold for the monaural signal in the right ear is redetermined. It is typically found under this condition, termed NO-Sm, that the intensity of the sinusoid must be decreased by about $9 \mathrm{~dB}$ (at $300 \mathrm{cps}$ ) in order to reestablish the masked threshold. This difference in signal intensity of $9 \mathrm{~dB}$ is the masking-level difference, or MLD, of NO-Sm relative to Nm-Sm. Although the magnitude of the MLD is a function of the frequency of the signal, the effect is easily measured over the range from 200 to $1000 \mathrm{cps}$.

It might be conjectured that the MLD obtained for NO-Sm is due simply to a facilitative, or synergistic, effect from the noise in the nonsignal ear. That this is not the case is evidenced by the fact that if the two noises are uncorrelated (NU-Sm), the detectability of the signal is the same as with the monotic condition.
The symbols used in the current literature on masking-level differences are sometimes confusing and it may help the reader to understand this nomenclature if the symbols employed in this paper to describe the various interaural conditions are all defined at this time. The symbols used are: $\mathrm{N}$ for the masking noise, $\mathrm{S}$ for the sinusoidal signal, 0 for an interaural phase shift of zero degrees, $\pi$ for an interaural phase shift of $180 \mathrm{deg}$, $U$ for an uncorrelated noise, and $\mathrm{m}$ for a monaural presentation of the stimulus. Thus, Nm-Sm represents the monotic listening condition (a reference condition for the measurement of MLDs); NU-Sm, the noise in one ear is uncorrelated with that in the other, and the signal is monaural (MLD $=0 \mathrm{~dB}$ ); NO-SO, the interaural phase shift of the binaural noise and of the binaural signal is zero degrees (MLD $=0 \mathrm{~dB}$ ); NO-Sm, the interaural phase shift of the binaural noise is zero degrees and the signal is monaural (MLD $=9 \mathrm{~dB}) ; \mathrm{N} \pi-\mathrm{S} 0$, the interaural phase shift of each component of the binaural noise is $180 \mathrm{deg}$ and that of the binaural signal is zero degrees $(M L D=13 \mathrm{~dB}$ ); NO-S $\pi$, the interaural phase shift is zero degrees for the noise and is $180 \mathrm{deg}$ for the signal $(M L D=15 \mathrm{~dB})$

It should be noted that although a "release from masking" results under certain binaural stimulus conditions, there are other binaural situations in which the detectability of a signal is the same as that for the monotic condition. Consequently, the conditions of binaural listening may be classified into MLD conditions and nonMLD conditions.

There are two functional relations that are particularly informative in the determination of the sensitivity of a sensory system. One of the relations is called the receiver-operating characteristic, or ROC. The ROC shows the relation between the O's proportion of "correct acceptances" and his proportion of "incorrect acceptances" with the stimulus conditions (signal and noise) held at constant average values. There is now little question that a measure of sensitivity derived from an ROC is superior to a measure of the classical threshold (Green \& Swets, 1966). ROCs have been determined by a number of investigators for MLD conditions and for nonMLD conditions (e.g., Egan et al, 1959; Watson et al, 1964; Green \& Swets, 1966; Schulman \& Mitchell, 1966; Markowitz \& Swets, 1967; Emmerich, 1968).

A second functional relation that is useful in the study of the sensitivity of a system is called the psychometric function. In the context of signal detection and MLDs, such a function shows the relation between the energy of the signal and some measure of performance, such as the percentage of correct decisions. With some classical techniques, only one point on such a function can be determined, namely, the "masked absolute threshold." The measurement of the detectability of the signal at different values of signal energy obviously has the potential of providing more information about the nature of the process of detection than that provided by the measurement of a single value.

The present paper is concerned only with a comparison of psychometric functions for MLD conditions with those for nonMLD conditions. If the detection of the signal is based either upon a different method of signal processing or upon a different aspect of the input, it would not be surprising if the form of the psychometric functions were different under these two types of conditions. However, previous research (Egan et al, 1961; Egan et 

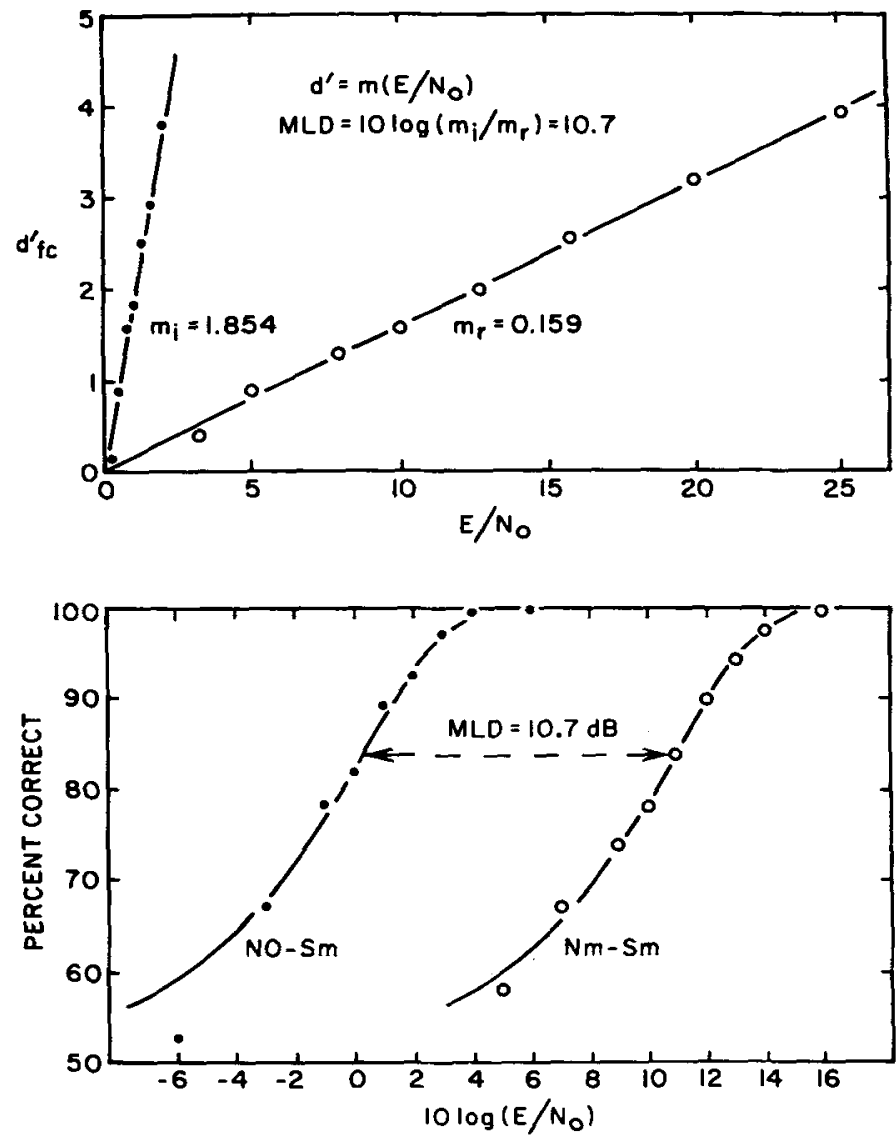

Fig. 1. These two graphs illustrate the transformation of the equation $d^{\prime}=m\left(E / N_{o}\right)^{k}$ into per cent correct plotted against decibels. The upper panel shows the data for one listener, $S 3$, obtained under two interaural conditions of listening, $\mathrm{Nm}-\mathrm{Sm}$ and NO-Sm. The exponent $k$ of the power function (Eq. 1) is the same for both interaural conditions so that the MLD is independent of the particular level of performance chosen for the definition of the MLD. The lower panel shows the same data as the upper panel, but replotted in the form per cent correct against decibels. When Eq. 1 is so transformed, the curve in the lower panel is simply displaced horizontally as $m$ is changed from one value to another. The conditions under which these data were secured are described in connection with Fig. 8.

al, 1965) had indicated that the psychometric functions for a variety of MLD and nonMLD conditions had nearly the same form, and the results apparently could be summarized by the following equation:

$$
d^{\prime}=m\left(E / N_{o}\right)^{k}
$$

In this equation, $d^{\prime}$ is a criterion-free measure of the $O$ 's performance, $E$ is the energy of the signal, $N_{o}$ is the noise power per unit bandwidth, and $m$ and $k$ are constants for a particular psychometric function. The results to be presented in this paper show that Eq. 1 provides a good fit to detection data; furthermore, the results suggest that $\mathbf{k}$ may be considered a constant for a particular $O$ and that $m$ depends upon the in teraural condition. When $\mathrm{k}$ is the same for the various interaural conditions, then it may be shown that

$$
\operatorname{MLD}=(10 / k) \log \left(m_{i} / m_{r}\right),
$$

where $m_{i}$ is the constant in Eq. 1 required for the interaural condition, $i$, and $m_{r}$ is the corresponding constant for the reference condition, $\mathrm{Nm}-\mathrm{Sm}$. Consequently, if $\mathrm{k}$ is constant, the magnitude of the MLD is independent of the level of performance chosen to define that MLD.

The psychometric function described by Eq. 1 can be plotted on a number of interesting coordinate systems. The most obvious, of course, is that shown in the upper panel of Fig. 1, where two psychometric functions are plotted as $d^{\prime}$ against $\left(E / N_{o}\right)$. The value of $k$ is 1.0 for both conditions of listening. When $k$ is 1.0 , it is fairly easy to compute the MLD from such a plot. However, many Os require a value of $\mathrm{k}$ larger than 1.0 , sometimes as large as 2.0. That is, when plotted in this space, their functions show considerable curvature. Consequently, it is difficult to estimate independently $\mathrm{k}$ and $\mathrm{m}$ of $\mathrm{Eq} .1$ for such $\mathrm{Os}$ on this coordinate system. In such instances, the constants, $k$ and $m$, may be more easily estimated after a transformation of the coordinates.

In the lower panel of Fig. 1 , the coordinates $d^{\prime}$ and $\left(E / N_{o}\right)$ have been transformed to per cent correct and $10 \log \left(E / N_{0}\right)$, respectively. This transformation of $\mathrm{d}^{\prime}$ was accomplished by consulting a table for the area under the normal curve corresponding to $d^{\prime} / 2$. (This area is known as maximum proportion correct, $\mathrm{P}(\mathrm{C})_{\mathrm{m}}$, and its characteristics are discussed in Egan, 1965, and in Appendix III of Green \& Swets, 1966.) When the data are replotted in this way, it is a simple matter to estimate $k$ and $m$ independently. This is so because various values of $m$ correspond to simple horizontal displacements of the psychometric function, and the value of $k$ is reflected in the steepness of the curve. Usually Eq. 1 fits the data for a single $O$ quite well over the range corresponding to 65 to $95 \%$ correct. (Equation 1 fits the data shown in Fig. 1 somewhat better than for the typical listener.) Figure 2 shows how various values of $k$ affect psychometric functions derived by this transformation of Eq. 1 . The data for nearly all Os may be fitted quite well with a value of $\mathrm{k}$ somewhere between 1.0 and 1.5. This graph illustrates that if $\mathrm{k}$ had quite different values for different MLD conditions, then the MLD would depend upon the level of performance chosen for the definition of that MLD. (It should be noted that the functions shown in Fig. 2 are not normal ogives.)

If the coordinates of Eq. 1 are transformed into $\log \mathrm{d}^{\prime}$ and $10 \log \left(E / N_{0}\right)$, as shown in Fig. 3 , the values of $k$ and $m$ may be estimated as the constants of a "best-fitting" straight line. 5 It

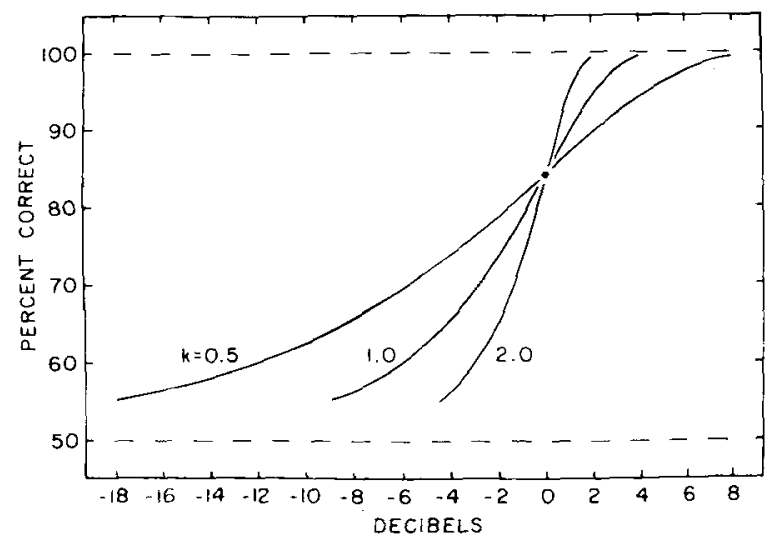

Fig. 2. These curves show how the slope of the psychometric function changes with changes in the value of $\mathrm{k}$. The three curves have been tied together at $84 \%$. For the simple tasks of detection considered here, data for nearly all listeners may be reasonably fitted by values of $k$ between 1.0 and 1.5. The function for $k=1.0$ requires approximately $11 \mathrm{~dB}$ to range from 55 to $95 \%$ correct. 


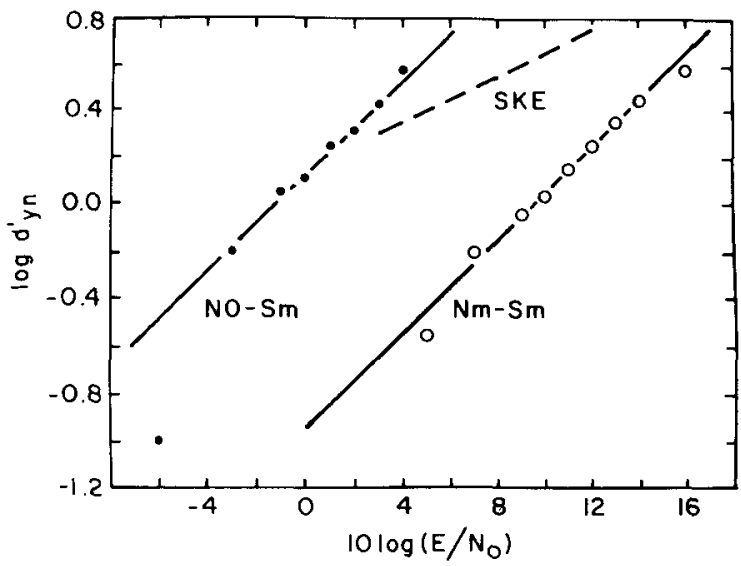

Fig. 3. These functions show the same data as those plotted in Fig. 1. The values of $d^{\prime}$ have been transformed from forced-choice to yes-no, $\mathbf{d}_{f \mathrm{c}}^{\prime}=\sqrt{2} \mathrm{~d}_{\mathrm{y} \mathrm{n}}^{\prime},($ Elliott, 1959). The straight lines are based upon the functions shown in Fig. 1. The lowest point for N0-Sm was ignored. The dashed curve shows the performance of an ideal $O$ for the situation in which the signal is the same on each trial, is known to the $O$ exactly, and is presented over a single channel.

should be noted, however, that such estimates are strongly biased by the effects of sampling errors at the extreme values of $d^{\prime}$; small sampling errors at extreme values of $\mathrm{d}^{\prime}$ result in large errors in $\log \mathrm{d}^{\prime}$. Furthermore, when an O performs perfectly, or when his performance is at the chance level, the corresponding values of $\log \mathrm{d}^{\prime}$ do not exist. With the coordinates of per cent correct and decibels, however, such data do have a meaningful graphical representation.

Some investigators (Tanner, 1959; Jeffress, 1964) choose to plot $d^{\prime}$ against $\left(2 \mathrm{E} / \mathrm{N}_{\mathrm{o}}\right)^{1 / 2}$. One of the most important cases of signal detection theory concerns the detection of a signal that is known exactly (SKE) by an ideal $O$. If it is assumed that this signal is presented in "Fourier-series, band-limited, white Gaussian noise," then, for this idealized case, the psychometric function has the form: $d^{\prime}=\left(2 E / N_{o}\right)^{1 / 2}$. Figure 4 shows the data of Fig. 1 for $\mathrm{Nm}-\mathrm{Sm}$ replotted on a coordinate system of $\mathrm{d}_{\mathrm{yn}}^{\prime}$ against $\left(2 \mathrm{E} / \mathrm{N}_{\mathrm{O}}\right)^{1 / 2}$ (Note 5$)$. Also included are two functions for the ideal $O$, one with the signal known exactly (SKE), and one with the signal known except for phase (SKS).

One of the more familiar ways of displaying a psychometric function is the following one. The hit rate, $P(S \mid s)$, is plotted against a variable related to signal energy, e.g., $10 \log \left(E / N_{\circ}\right)$. Typically, the ordinate is called the "probability of detection," the "probability of seeing," or the "probability of hearing," but the parameter related to "false-alarms" is not specified. The implication of these various descriptions is that a given ordinate is the probability that the $O$ will detect the signal when it is presented. By now, the difficulty with these presentations should be clear. There is a closely parallel situation in the realm of statistical tests of hypotheses. It is well recognized that the power of a statistical test has meaning only when the probability of a Type I error is specified for that test. Similarly, in order to be meaningful, a particular value of the "probability of hearing" when the signal is presented must have associated with it a particular value of the "probability of hearing" when there is "nothing" to hear. If the probability of a false-alarm (Type I error) is included as a parameter of a function relating the hit-rate to the signal energy, then the data may be mapped into a typical ROC space. Some writers prefer this method of presentation of a family of psychometric functions, particularly when the false-alarm rates vary over an enormous range, e.g., $10^{-6}$ to $10^{-1}$. For example, see one of the most elegant and most complete presentations of signal detection theory (Wainstein \& Zubakow, 1962).

Four coordinate systems for a psychometric function have been illustrated in Figs. 1.4. Although each system has its particular advantages, the results of the experiments reported in this paper will be presented in the form: the percentage of correct decisions against decibels (lower panel of Fig. 1). The advantages of this coordinate system for the measurement of MLDs may be summarized as follows: (1) the values of the parameters $m$ and $k$ may be readily determined; (2) it is somewhat easier to estimate the magnitude of an MLD, since the MLD is simply the horizontal distance between the two psychometric functions; (3) when an $\mathrm{O}$ happens to achieve a level of performance that is either perfect or at chance, the data may still be used to help determine the position and the slope of the psychometric function; finally, (4) this coordinate system has the advantage of simplicity of interpretation.

The results of several experiments will now be presented. The salient features of the various procedures are described in the next section, and certain details such as the duration and the frequency of the signal are presented along with the results.

\section{PROCEDURE}

Two psychophysical methods were employed: (1) two-interval, forced choice, or 2IFC, and (2) single-interval forced choice, or yes-no. Each of these methods was employed in such a way that a measure of $\mathrm{d}^{\prime}$ could be secured. These methods have been described in detail elsewhere (Green \& Swets, 1966), and only the principal features will be indicated here.

With the 2IFC procedure, two observation intervals were
Fig. 4. Psychometric functions for the ideal $O$ and for a human $O$. The function labeled SKE is for the situation in which the signal is the same on each trial and is known exactly by the ideal $O$. The function denoted by SKS is for the situation in which the signal is known except for phase. The data are the same as those shown in Figs. 1 and 3.

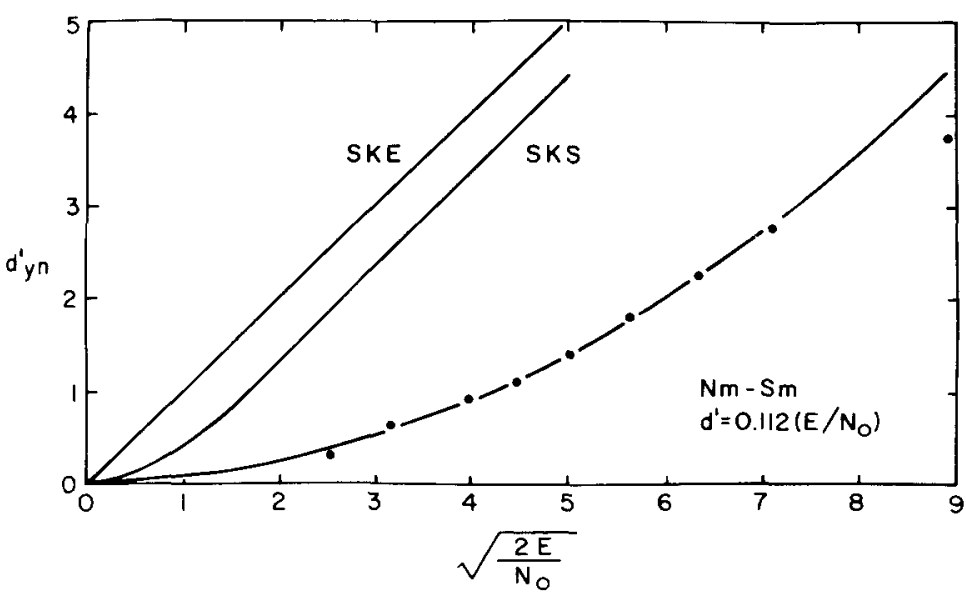


Fig. 5. Psychometric functions for five interaural conditions. Each point is based upon 180 or 240 trials, and each trial was presented simultaneously to all four listeners. The MLD for NO-Sm is about $9 \mathrm{~dB}$, and that for $\mathrm{NO}-\mathrm{S} \pi$ is about $14 \mathrm{~dB}$. Method: 2IFC. Signal: $500 \mathrm{cps,}$ 250 msec. Noise: $45 \mathrm{~dB} / \mathrm{cy}$. Value of $k: 1.0$ for each curve.

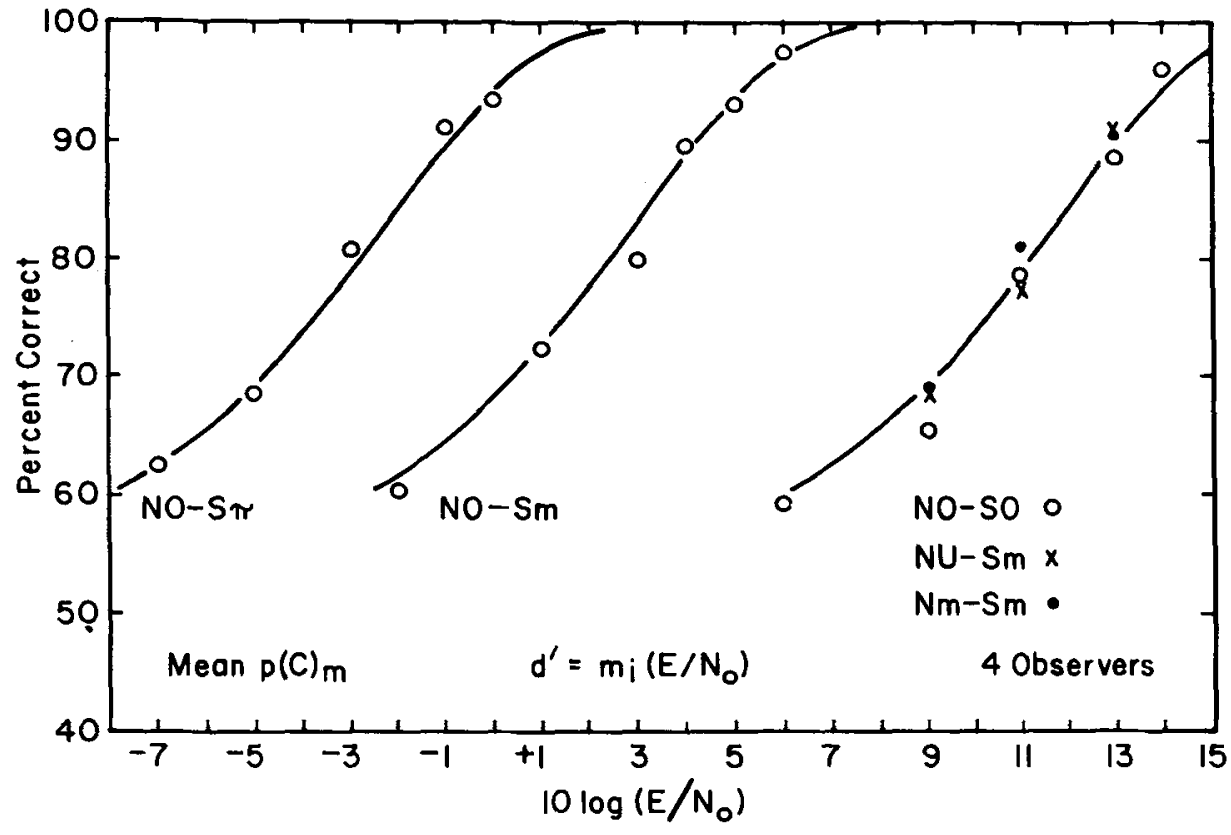

also given frequent "tone demonstrations" and practice blocks during each 1.5 -h session.

$0.5 \mathrm{sec}$ between them. On each trial, the signal was presented only once, and it occurred in either the first or the second interval with equal a priori probability. Thus, there were two types of trials, and the $O$ was required to respond with either first or second interval on each trial. A trial consisted of the following: warning light $(500 \mathrm{msec})$, pause $(500 \mathrm{msec})$, light for first observation interval $(250 \mathrm{msec})$, pause $(500 \mathrm{msec})$, light for second observation interval (250 msec), response interval $(2.5 \mathrm{sec})$, and feedback interval $(500 \mathrm{msec})$.

In the single-interval method, only one observation interval was indicated ner trial, and the signal, when presented, occurred during this interval. After each observation interval, the $\mathrm{O}$ was required to respond with either yes or no. The a priori probability of the occurrence of the signal was 0.5 . In all other respects, except for the response interval $(2.0 \mathrm{sec})$, the trials for the single-interval method were identical to those for 2IFC. In both methods, which of the two possible events occurred on a trial was indicated to the Os during the feedback interval at the end of each trial.

The occurrence of the signal in an observation interval with either of the two psychophysical methods was automatically programmed from a table of random numbers. Thus, the occurrence of the signal was determined by an "independenttrials process."

The bandwidth of the white noise was determined by the earphones (Permoflux Corporation, PDR-10), and this noise was present continuously throughout a block of trials. For all tests, the spectrum level of the noise was $45 \mathrm{~dB}$ re 0.0002 microbar. The signal was gated by a mercury-wetted relay without regard for phase at onset, and the (negligible) transients were determined by the response of the earphone. The members of a given listening crew were tested simultaneously, and the waveforms on a given trial were the same for all crew members. In all experiments, a single value of the signal energy was used for a given block of 60 or 80 trials.

The data for the different interaural conditions were collected in an order that was counterbalanced over sessions. All members of the listening crews were young adults with clinically normal hearing; they were paid for their services. All Os were given extensive training before the experiment began, and they were

\section{RESULTS}

Figure 5 shows the results obtained in an experiment previously reported (Egan, 1965). Five interaural conditions are represented, and each of the three smooth curves is the transform of Eq. 1 with $k=1.0$. The numerical values of the MLDs are typical of those obtained with modern psychophysical methods. The data shown in Fig. 5 were secured in experiments that were designed primarily for the measurement of MLDs. Consequently, the earlier report did not include the psychometric functions shown in Fig. 5. However, these functions seemed to be so similar that two experiments were specifically designed to determine the form of the psychometric function for various interaural conditions.

In the first of the two additional experiments, psychometric functions were determined for two interaural conditions, NO-SO and NO-S $\pi$. Performance was measured at six values of signal energy, and 640 trials were conducted at each of these levels in the hope that the details of the functions would be well established for each of the four Os. Figure 6 shows the results. The data for each $\mathrm{O}$ are referred to a separate horizontal axis, and the values along these axes are correct for the condition, NO-SO. However, for the NO-S $\pi$ conditions each point is displaced horizontally to the right by an amount equal to the MLD for that $\mathrm{O}$. The smooth curves are based on the transform of Eq. 1, and the agreement with the data is considered to be fair. Figure 7 shows the results when the data of Fig. 6 are averaged. Again, the horizontal axis is correct only for NO-SO; for NO-S $\pi$, each point is displaced horizontally to the right by $15.6 \mathrm{~dB}$. The smooth curve is based on the transform of Eq. 1 with $\mathrm{k}=1.6$, and the curve is a good fit to the data.

The extensive data of this first additional experiment were analyzed in various ways. For the data based upon the experiment proper, there were insignificant effects regarding (1) the level of performance as a function of practice, and (2) the level of performance depending upon an ascending or a descending order of intensities of the signal during a session.

In the second of the two additional experiments, psychometric functions were determined for three interaural conditions, 


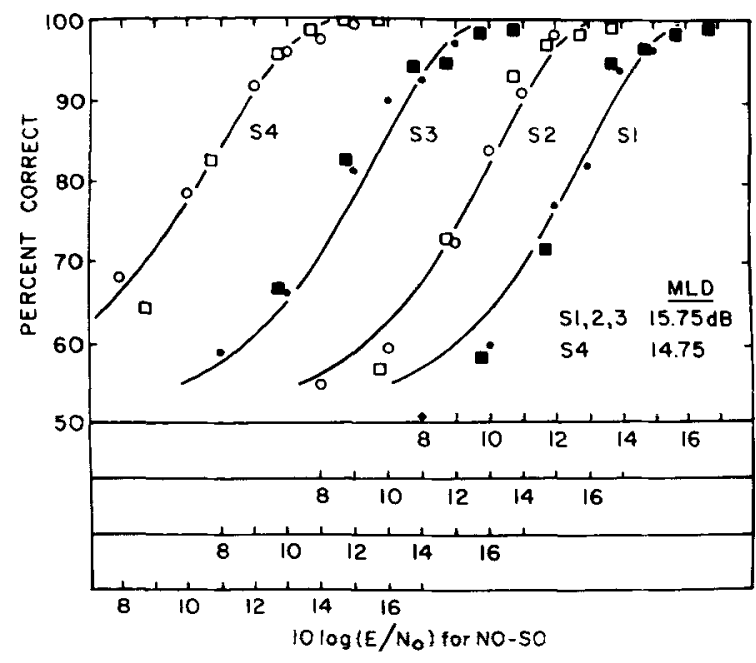

Fig. 6. Psychometric functions for each of four Os. There were two interaural conditions, NO-SO (circles) and NO-S $\pi$ (squares). Each of the four sets of data has its own horizontal axis. The values along the horizontal axis are correct for the condition NO-SO; however, for the condition NO-S $\pi$ the abscissa of each point has been increased by $15.75 \mathrm{~dB}$ for $S 1, S 2$, and $S 3$, and by $14.75 \mathrm{~dB}$ for $\mathrm{S} \mathrm{4}$. The value of $\mathrm{k}$ in $\mathrm{Eq} .1$ is 1.5 for $\mathrm{S} 1, \mathrm{~S} 2$, and $S 3$, and it is 1.25 for $S 4$. Method: $2 I F C$. Signal: $390 \mathrm{cps}$, $250 \mathrm{msec}$. Noise: $45 \mathrm{~dB} / \mathrm{cy}$.

$\mathrm{Nm}-\mathrm{Sm}, \mathrm{NO}-\mathrm{Sm}$, and NO-S $\pi$. In a total of 24 sessions, performance was measured at nine values of signal energy for each of the three interaural conditions. In a particular session, data were collected for only one interaural condition, and one block of 60 trials was devoted to each of the nine signal levels. For each of the three interaural conditions, the signal level was progressively decreased during the session for four sessions, and it was increased during the other four sessions. Figure 8 shows the results for each $O$ separately. Again, a single value of $k$ in Eq. 1 was used for the three curves in a given panel. (The data shown in Fig. I were taken from S 3.) Table 1 shows the values of $10 \log \left(\mathrm{E} / \mathrm{N}_{\mathrm{O}}\right)$ required for $84 \%$ correct detections (2IFC) for each of the three interaural conditions. 6 The MLDs are also tabulated.

Figure 9 shows the results when the data of Fig. 8 are averaged. The horizontal axis is correct only for $\mathrm{Nm}-\mathrm{Sm}$; for N0-Sm and for $\mathrm{NO}-\mathrm{S} \pi$, each point is displaced by an amount corresponding to the magnitude of the MLD. Again, the fit of the curve to the data is considered to be quite good.

The psychophysical method used to secure the data shown in Figs. 5-9 was that called two-interval, forced choice, or 2IFC. For
Table 1

Values of $10 \log \left(\mathrm{E} / \mathrm{N}_{\mathrm{o}}\right)$ required for $84 \%$ correct detections (2IFC) for the data plotted in Fig. 8. The MLD's are also shown.

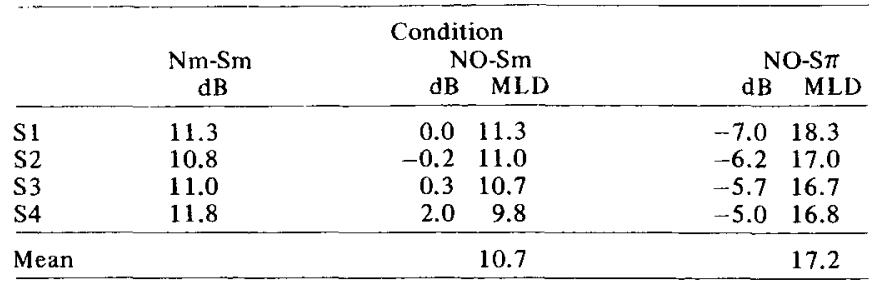

the final set of psychometric functions, the method was that called the single-interval, or yes-no method. Psychometric functions were obtained for each of three interaural conditions, $\mathrm{NO}-\mathrm{SO}, \mathrm{N} \pi$-SO, and NO-S $\pi$. Four signal levels were used for each condition, and six blocks of 80 trials each were conducted at each of these signal levels. Figure 10 shows the average psychometric functions, and the transform of Eq. 1 with $k=1.0$ is a good fit to the data. These functions were determined in an experiment designed for other purposes (McFadden, 1966). Figure 10 indicates that Eq. 1 may be used to describe the forms of the psychometric functions for data secured with the yes-no method as well as with the 2IFC method.

\section{DISCUSSION}

Equation 1 is purely an empirical relation, but it has the advantage of simplicity. The transformation of the variables of Eq. 1 to per cent correct and decibels makes it relatively easy to compare the forms of functions obtained from different Os and with different stimulus conditions. In this paper we have reported data that support the notion that the form of the psychometric function for detection is the same for various interaural conditions of listening. For a particular $O$, the same value of $k$ may be used to describe psychometric functions that are displaced from each other by as much as $17 \mathrm{~dB}$.

The measurement of the detectability of the signal at several values of $\left(E / N_{0}\right)$ provides more information about the nature of the process of detection than that provided by the measurement of a masked absolute threshold. Let us repeat two points. First of all, because the same value of $k$ in Eq. 1 may be used for each interaural condition, any level of performance may be selected to determine MLDs. Secondly, it is possible to compare levels of performance for different interaural conditions at a single value of signal energy. For example, consider the function for $\mathrm{Nm}-\mathrm{Sm}$ shown in Fig. 5. With a value of $10 \log \left(E / N_{0}\right)$ of $6 \mathrm{~dB}$, the per cent correct is about 60 , a level of detectability below the "classical threshold." When the same noise is added to the other ear, so that the condition becomes NO-Sm, the level of
Fig. 7. Average psychometric functions for the data of Fig. 6. Each point is a mean based upon four listeners. The horizontal axis is correct only for NO-SO; for NO-S $\pi$ each point is displaced horizontally to the right by $15.6 \mathrm{~dB}$. The smooth curve is based upon the transform of Eq. 1 with $k=1.6$.

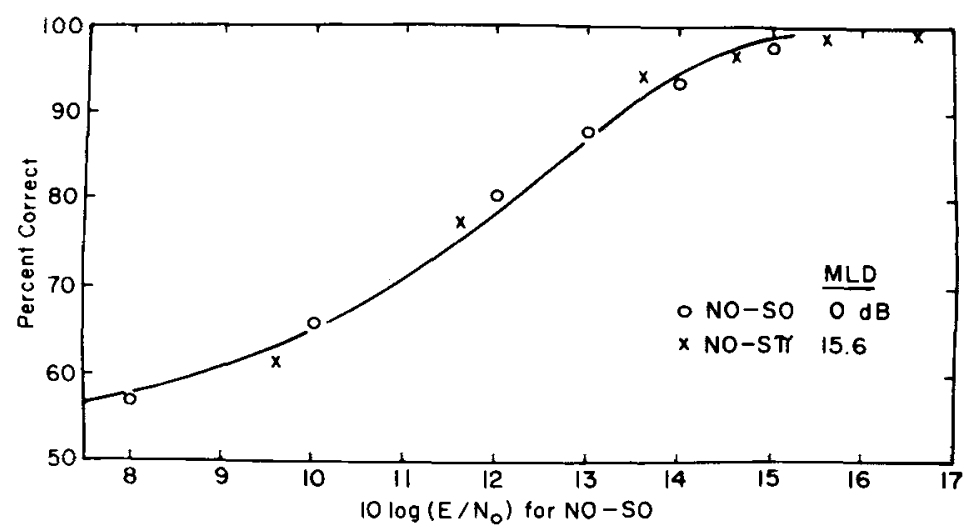



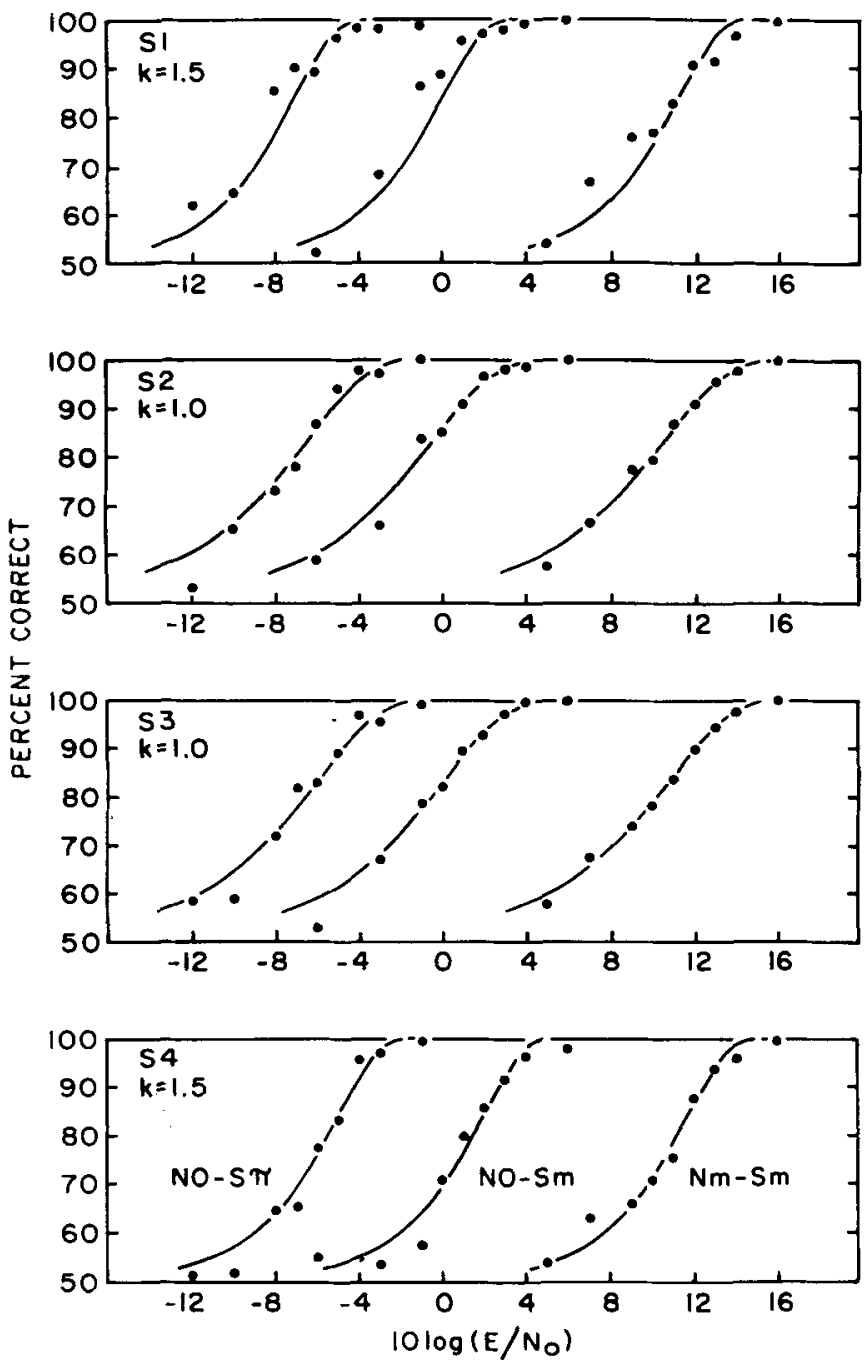

Fig. 8. Psychometric functions for three interaural conditions. The results are shown for each $O$ separately. Each point is based upon 480 trials. The smooth curves are based on the transform of Eq. 1, and a single value of $k$ was used for the three curves in a given panel. Method: 2IFC. Signal: $390 \mathrm{cps}, 250 \mathrm{msec}$. Noise: $45 \mathrm{~dB} / \mathrm{cy}$.

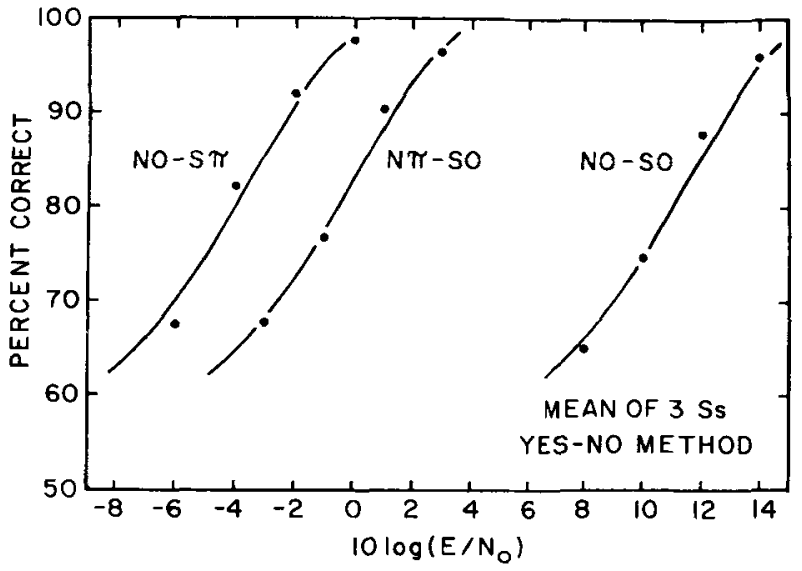

Fig. 10. Average psychometric functions for three interaural conditions obtained by the single-interval, or yes-no method. The smooth curves are based upon the transform of Eq. 1 with $k=1.0$. Method: single interval. Signal: $400 \mathrm{cps}, 125 \mathrm{msec}$. Noise: $45 \mathrm{~dB} / \mathrm{cy}$.

performance jumps to about $97 \%$. That still popular theory of monaural masking, called the "line-busy effect," is hardly tenable in the face of such results, and Hirsh (1948) tentatively drew this conclusion. Under monaural conditions of masking, a signal that is well below the classical threshold has an effect in the peripheral nerve, and under appropriate binaural conditions, this monaural signal may be readily ex tracted from the noise.

Green (1966b) has also secured data using a large range of signal energies for each of several interaural conditions, and he has also concluded that the forms of the psychometric functions are very similar. The particular psychometric function that Green fitted to his data was that for the energy detector; such a psychometric function corresponds to a $\mathbf{k}$ of 1.0 in Eq. 1. However, Green notes that some of his data might have been fitted better by values of $k$ greater than 1.0 . He also points out that this fact cannot be attributed to effects of averaging either across sessions or across Os, for if there were systematic changes in the position of a psychometric function, averaging would have the effect of reducing the slope, not increasing it.

Caution must be exercised when interpreting the fact that the psychometric functions for MLD and nonMLD conditions are of the same form, because the form of such functions alone is not enough information to permit strong conclusions about the

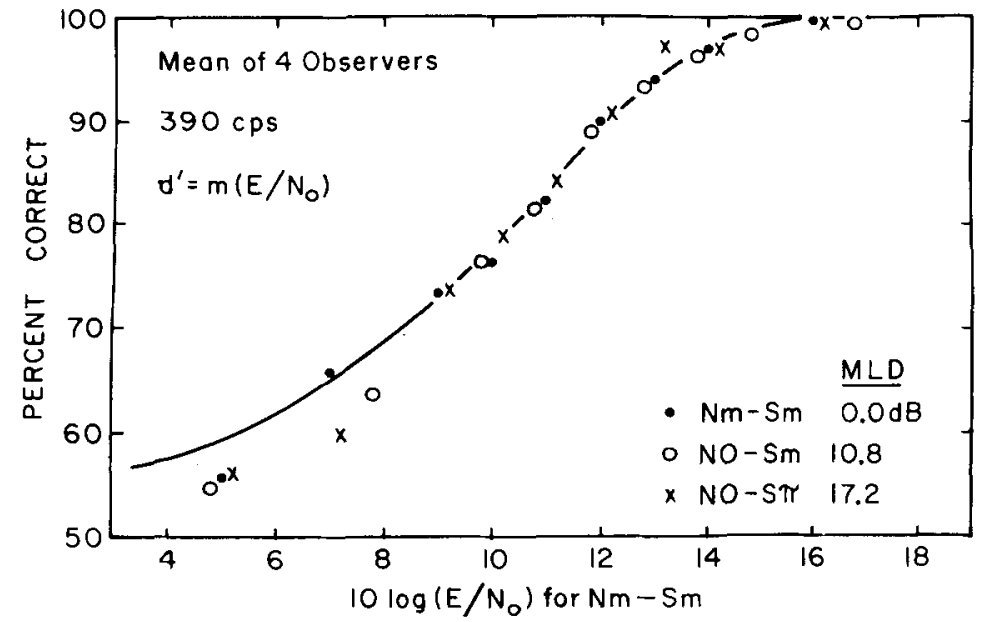

Fig. 9. Average psychometric functions for the data of Fig. 8. Each point is a mean based upon four Os. The horizontal axis is correct only for Nm-Sm. For NO-Sm, each point is displaced horizontally to the right by $10.8 \mathrm{~dB}$, and for $\mathrm{NO}-\mathrm{S} \pi$, by $17.2 \mathrm{~dB}$. The smooth curve is based upon the transform of Eq. 1 with $\mathbf{k}=1.0$. 
nature of the underlying mechanisms. The form of the psychometric function is determined by several independent factors, such as the bandwidth of the "internal filter," the method of processing, and the aspect of the input being processed. Consequently, if the forms of two functions were quite different, it would not be possible to state with certainty which one of these factors was responsible for the difference. Similarly, if the forms of two functions were the same, one could not conclude anything definite about a particular factor because certain factors might have compensating effects upon the form of the functions.

Several models of the monaural detection process that predict both the form and the location of the psychometric function have been proposed (McGill, 1968; Jeffress, 1968). Most of these models lead to functions with slopes corresponding to values of $\mathrm{k}$ which are equal to or less than 1.0. On the other hand, results from several laboratories are in agreement that values of $k$ greater than 1.0 are frequently required to fit the data for an individual listener. This does not necessarily mean that the basic assumptions of these models are incorrect. With a few minor changes, one of these models might result in better fits to the data. Alternatively, it is possible that a refinement in our experimental procedures might yield data which are in better agreement with some of these models.

\section{REFERENCES}

BLODGETT, $\mathrm{H}, \mathrm{C}$ JEFFRESS, L A. \& TAYLOR, $\mathrm{R}, \mathrm{W}$. Relation of masked threshold to signal duration for various interaural phase combinations. American Journal of Psy chology, 1958, 71, 283-290.

DURLACH, N. I. Equalization and cancellation theory of binaural masking-level differences. Journal of the Acoustical Society of America, $1963,35,1206-1218$.

EGAN, J, P. Masking-level differences as a function of interaural disparities in intensity of signal and of noise. Journal of the Acoustical Society of America, 1965, 38, 1043-1049.

EGAN, J. P., GREENBERG, G. Z., \& SCHULMAN, A. I. Operating characteristics determined by binary decisions and by ratings. Journal of the Acoustical Society of America, 1959, 31, 768-773.

EGAN, J. P., GREENBERG, G. Z., \& SCHULMAN, A. I. Interval of time uncertainty in auditory detection. Journal of the Acoustical Society of A merica, 1961, 33, 771-778.

IGAN, J. P., LINDNER, W. A., \& Mcl ADDEN, D. Masking-level differences and the form of the psychometric function. Joumal of the Acoustical Society of America, 1965, 37, 1181. (Abstract)

IELLIOTT. P. B. Tables of $d^{\prime}$. Electronics Defense Group Technical Report No. 97. University of Michigan, 1959.

EMMERICH, D. S. Receiver-operating characteristics determined under several interaural conditions of listening. Joumal of the Acoustical Socjety of America. 1968, 43, 298-307.

GREEN, D. M. Signal-detection analysis of equalization and cancellation model. Joumal of the Acoustical Society of America, 1966a, 40, 833-838.

GREEN. D. M. Interaural phase effects in the masking of signals of different durations. Journal of the Acoustical Society of America, $1966 \mathrm{~b}, 39,720-724$.

GREEN, D. M., \& SWETS, J. A. Signal detection theory and psychophysics. New York: Wiley, 1966.

HIRSH, I. J. The influence of interaural phase on interaural summation and inhibition. Journal of the Acoustical Society of America, 1948, 20, 536-544.

HIRSH, I. J., \& WEBSTER, F. A. Some determinants of interaural phase effects. Journal of the Acoustical Society of America, 1949, 21 , 496-501.
JEFFRESS, L. A. Stimulus-oriented approach to detection. Joumal of the Acoustical Society of America, 1964, 36, 766-774.

JEFFRESS, L. A. Mathematical and electrical models of auditory detection. Journal of the Acoustical Socicty of America, 1968, 44, 187-203.

JEFFRESS, L. A., BLODGETT, H. C., \& DEATHERAGE, B. H. The masking of tones by white noise as a function of the interaural phases of both components. I. 500 cycles. Joumal of the Acoustical Society of America, 1952, 24, 523-527.

JEFFRESS, L. A., BLODGETT, H. C., SANDEL, T. T., \& WOOD, C. L., III. Masking of tonal signals. Journal of the Acoustical Society of America, 1956, 28, 416-426.

LANGFORD, T. L., \& JEFFRESS, L. A. Effect of noise crosscorrelation on binaural signal detection. Journal of the Acoustical Society of America, 1964, 36, 1455-1458.

L.ICKLIDER, J. C. R. The influence of interaural phase relations upon the masking of speech by white noise. Journal of the Acoustical Society of America, 1948, 20, 150-159.

MARKOWITZ, J., \& SWETS, J. A. Factors affecting the slope of empirical ROC curves: Comparison of binary and rating responses. Perception \& Psychophysics, 1967, 2, 91-100.

MCF ADDEN, D. Masking-level differences with continuous and with burst masking noise. Journal of the Acoustical Society of America, 1966, 40, 1414-1419.

McGILL, W. J. Variations on Marill's detection formula. Journal of the Acoustical Society of America, 1968, 43, 70-73.

ROBINSON, D. E., \& JEFFRESS, L. A. Effect of varying the interaural noise correlation on the detectability of tonal signals. Journal of the Acoustical Society of America, 1963, 35, 1947-1952.

SCHULMAN, A. I., \& MITCHELL, R. R. Operating characteristics from yes-no and forced-choice procedures. Journal of the Acoustical Society of America, 1966, 40, 473-477.

TANNER, W. P., JR. Graphical presentation of data in the framework of the theory of signal detectability. Journal of the Acoustical Society of America, 1959, 31, 243-244.

WAINSTEIN, L. A., \& ZUBAKOW, V. D. Extraction of signals from noise. Englewood Cliffs, N.J.: Prentice-Hall, 1962.

WATSON, C. S., RILLING, M. E., \& BOURBON, W. T. Receiver-operating characteristics determined by a mechanical analog to the rating scale. Journal of the Acoustical Society of America, 1964, 36, 283-288.

\section{NOTES}

1. This research was reported at the 69 th meeting of The Acoustical Society of America, June 2-5, 1965. These studies were supported in part by the Air Force Office of Scientific Research. The apparatus was constructed with funds granted by the National Science Foundation.

2. Present address: Department of Psychology, University of Washington, Seattle, Washington 98105.

3. Now at the California State College, Fullerton

4. Now at the University of Texas, Austin.

5. The same data were used for Figs. 1, 3, and 4, but for various reasons they appear as $d_{y n}^{\prime}$ in Figs. 3 and 4 , and as $d_{f c}^{\prime}$ in Fig. 1. The transformation is simply $\sqrt{2} \mathrm{~d}_{\mathrm{yn}}^{\prime}=\mathrm{d}_{\mathrm{fc}}^{\prime}$.

6. When the focus of investigation is upon the properties of a sensory system, it is desirable to eliminate two well-established potential sources of error: (1) the effects of the O's criterion (sometimes called "response bias"), and (2) the effects of sequential dependencies over trials. The results of the most pertinent experiments indicate that with trained Os the effects of sequential dependencies are negligible when $d^{\prime}>0.5$. We rather arbitrarily chose $\mathrm{d}^{\prime} \mathrm{fc}_{\mathrm{c}}=\mathbf{2 . 0}$, or $84 \%$ correct.

(Accepted for publication March 17, 1969.) 